contamination with enterococci and skin organisms: implications for surveillance definitions of primary bloodstream infections. Am J Infect Control 2011;39:436-438.

4. Weinstein MP, Towns ML, Quartey SM, et al. The clinical significance of positive blood cultures in the 1990s: a prospective comprehensive evaluation of the microbiology, epidemiology and outcome of bacteremia and fungemia in adults. Clin Infect Dis 1997;24:584-602.

5. Weinstein MP, Reller LB, Murphy JR, Lichtenstein KA. The clinical significance of positive blood cultures: a comprehensive analysis of 500 episodes of bacteremia and fungemia in adults. 1 . Laboratory and epidemiologic observations. Rev Infect Dis 1983; 5:35-53.

6. Everts RJ, Vinson EN, Adholla PO, Reller LB. Contamination of catheter-drawn blood cultures. J Clin Microbiol 2001;39:33933394.

\section{Reply to Freeman et al}

To the Editor-We thank Freeman et $\mathrm{al}^{1}$ for their comments and share their concerns that some enterococcal blood isolates classified as central line-associated bloodstream infections (CLABSIs) by current definitions may be contaminants. The potential for Enterococcus to be a contaminant in blood cultures was recognized at least 40 years ago. ${ }^{2}$ Our study compared the microbiology of National Healthcare Safety Network (NHSN)-defined CLABSIs in patients with and without neutropenia following chemotherapy, and we proposed a list of organisms for exclusion from the CLABSI definition in the setting of neutropenia. ${ }^{3}$ Although our study was not designed to assess the possibility that blood cultures growing a single Enterococcus species are contaminants, we queried our database to answer most of the questions posed by Freeman and colleagues.

Of the 52 patients in our study with CLABSIs that included enterococci, $26(50 \%)$ had more than 1 positive blood culture with the same enterococcal species, strongly suggestive of true infection. Of the 26 patients with a single blood culture yielding Enterococcus species, 5 (9.6\% of the total) had 1 or more typical skin contaminants defined by the NHSN in the same blood culture bottle as the Enterococcus species; all of these cultures were obtained through a central line. Overall, 40 patients (77\%) had at least 1 positive culture growing Enterococcus species drawn through a central line; $23(58 \%)$ of 40 also had the Enterococcus species isolated from a percutaneous culture.

We stratified this analysis by whether the enterococcal bacteremia occurred in the setting of neutropenia. Neutropenic patients were more likely to have multiple enterococcal isolates (10 [67\%] of 15 with multiple isolates) and were unlikely to have blood isolates suspicious for being a contaminant. Of the 5 patients with 1 enterococcal isolate, 3 died and 1 was transferred to a hospice within 10 days of the positive culture, suggesting that the bacteremia was significant. At least 1 enterococcal isolate was obtained from a central line-drawn blood culture in all 15; in 6 patients, the organism was also isolated from a percutaneously drawn blood culture.

Sixteen of the 37 nonneutropenic patients had multiple isolates of Enterococcus. Among the nonneutropenic patients, $25(68 \%)$ had at least 1 positive blood culture obtained through a central line, 12 of whom had blood cultures obtained through a central line and by peripheral venipuncture. There were 6 nonneutropenic patients who had a single enterococcal isolate where culture results and clinical context suggested that the Enterococcus was a contaminant. In all 6 patients, the enterococcal isolate was line drawn. These cases included 2 patients with 1 line-drawn blood culture bottle growing Enterococcus species and a common contaminant with another set showing no growth. As mentioned by Freeman and colleagues, this situation suggests that the Enterococcus does not represent true bacteremia. Interestingly, there were 4 patients with 1 line-drawn enterococcal isolate ( 1 with a common contaminant as well), each of whom had 2 blood cultures growing Staphylococcus aureus; in all 4 patients, catheter tip cultures grew more than 15 colonies of $S$. aureus. While these latter 4 cases would not change our CLABSI rate, they do highlight the potential of Enterococcus to be a contaminant.

Although the number of enterococcal infections in our series is small, these additional data reinforce our findings that enterococcal bloodstream infections are overrepresented in persons with neutropenia. If we censor the 6 enterococcal isolates suspected of being contaminants (all in nonneutropenic patients), the relative proportion of CLABSIs containing an Enterococcus species would be further skewed toward those with neutropenia (23\% in the neutropenic group vs $13 \%$ in the nonneutropenic group; $P=.05$ ).

We agree with Freeman and colleagues that determining the significance of enterococcal blood isolates is vexing, particularly with blood cultures obtained through central venous catheters.

\section{ACKNOWLEDGMENTS}

Potential conflicts of interest. All authors report no conflicts of interest relevant to this article. All authors submitted the ICMJE Form for Disclosure of Potential Conflicts of Interest, and the conflicts that the editors consider relevant to this article are disclosed here.

\section{Chad Robichaux, BA; ${ }^{1}$ Jesse T. Jacob, $\mathrm{MD}^{2}$ James P. Steinberg, $\mathbf{M D}^{2}$}

Affiliations: 1. Emory Healthcare, Atlanta, Georgia; 2. Division of Infectious Diseases, Department of Medicine, Emory University School of Medicine, Atlanta, Georgia.

Address correspondence to James P. Steinberg, MD, Medical Office Tower, 5th Floor, 550 Peachtree Street NE, Atlanta, GA 30308 (jstei02@emory.edu). Infect Control Hosp Epidemiol 2013;34(7):763-764 
(C) 2013 by The Society for Healthcare Epidemiology of America. All rights reserved. 0899-823X/2013/3407-0020\$15.00. DOI: $10.1086 / 671075$

\section{REFERENCES}

1. Freeman JT, Anderson DJ, Sexton DJ. Enterococcus species and the central line-associated bloodstream infection surveillance definition: evaluating the importance of blood culture contamination. Infect Control Hosp Epidemiol 2013;34:762-763 (in this issue).

2. MacGregor RR, Beaty HN. Evaluation of positive blood cultures: guidelines for early differentiation of contaminated from valid positive cultures. Arch Intern Med 1972;130:84-87.

3. Steinberg JP, Robichaux C, Tejedor SC, Reyes MD, Jacob JT. Distribution of pathogens in central line-associated bloodstream infections among patients with and without neutropenia following chemotherapy: evidence for a proposed modification to the current surveillance definition. Infect Control Hosp Epidemiol 2013;34:171-175.

\section{Epidemic Keratoconjunctivitis Outbreak in a Closed Psychiatric Ward}

To the Editor-Having read the article by Fukuta and Muder on infectious outbreaks in psychiatric facilities, we would like to share our experience with an outbreak of epidemic keratoconjunctivitis (EKC) that occurred in a closed psychiatric ward of our hospital. The Department of Psychiatry is situated in a pavilion with 3 floors and 5 wards. The affected ward is a closed unit with 26 beds in 12 double rooms and 2 single rooms (each with its own bathroom), a nurses' station, a dining room, and a common room for group activities. At the point of notification, 22 beds were occupied.

The first notification of the outbreak reported 5 cases of adenoviral EKC. The index case was traced; symptoms in this patient started 13 days before the notification.
We recommended a set of outbreak control measures based on general and hand hygiene and on appropriate disinfection of surfaces (Table 1), and we organized workshops on hand hygiene for all staff. However, within 18 days of the notification the number of affected patients rose to 11 , and 1 probable case was identified in a staff member. In light of such an increase in cases, we decided to restrict any further admissions and discharge as many patients as possible. When only 2 patients remained, an exhaustive cleaning of the whole ward was done. Usual activity was resumed the next day.

The epidemic curve was bimodal, with peaks in the first and third weeks of the outbreak. The last case of EKC was identified 34 days after the first notification and 47 days after the beginning of symptoms in the index case. Altogether, 13 cases of EKC were identified among patients of the closed unit. There were 2 suspected cases within this unit and 4 more in other units, none of which fulfilled the diagnostic criteria. The overall attack rate among patients was $22.4 \%$ $(13 / 58)$. Of the 6 workers who developed some symptoms, only 2 were identified as cases (a nursing assistant. and a cleaner). The attack rate among staff was $11.7 \%(2 / 17)$. No infection of patient visitors was noted.

EKC is a highly contagious disease and spreads very fast in hospital settings. The transmission probably occurred through direct person-to-person contact and use of common spaces. Implementation of preventive measures in a psychiatric ward is very difficult. The psychiatric pathologies of the patients that impeded strict adherence to hygiene rules, excessive physical activity, use of shared objects for group activities (eg, painting supplies), and especially direct physical contact all might have played a role in the spread of the disease. Patients could not be contained in their individual rooms, and preventing them from touching their itchy eyes was virtually impossible. Alcohol-based hand rub dispensers could not be installed because of the risk of ingestion, and

TABLE 1. Outbreak Control Measures

1. Intensification of hand hygiene in staff members

A. Distribution of individual bottles of alcohol-based hand sanitizer

B. Promotion of hand hygiene; use of gloves for eye care, and hand hygiene before and after the use of gloves

2. Improvement of patient hygiene

A. Promotion of hand hygiene

B. Removal of towels from bathrooms; use of disposable towels; removal of bath towels immediately after use

C. Daily change of clothes

D. Restriction of contact among patients as much as possible

3. Intensification of the cleaning measures

A. Use of quaternary ammonium-based cleaning products

B. Frequent cleaning in the zones of increased contact with hands: door handles, light switches, tables, etc

4. Individual rooms for patients with conjunctivitis or, if not possible, patient cohorting

5. Division of the common areas for separate use by patients with and without epidemic keratoconjunctivitis

6. Immediate notification of new cases to the Department of Preventive Medicine and Quality Management

7. Referral of any worker with suspicion of conjunctivitis to the Department of Occupational Health

8. Suspension of new admissions until the symptoms of affected patients disappear ${ }^{2}$

9. Closure of the ward for thorough cleaning

a Measures 8 and 9 were taken during the second peak of the outbreak. 